\title{
Laser-induced thermal-acoustic velocimetry with heterodyne detection
}

\author{
Stefan Schlamp \\ Graduate Aeronautical Laboratories, California Institute of Technology, Pasadena, California 91125
}

Eric B. Cummings

Sandia National Laboratories, Livermore, California 94551

Thomas H. Sobota

Advanced Projects Research Incorporated, La Verne, California 91750

Received September 20, 1999

\begin{abstract}
Laser-induced thermal acoustics (LITA) was used with heterodyne detection to measure simultaneously and in a single laser pulse the sound speed and flow velocity of $\mathrm{NO}_{2}$-seeded air in a low-speed wind tunnel up to Mach number $M=0.1$. The uncertainties of the velocity and the sound speed measurements were $\sim 0.2 \mathrm{~m} / \mathrm{s}$ and $0.5 \%$, respectively. Measurements were obtained through a nonlinear least-squares fit to a general, analytic closed-form solution for heterodyne-detected LITA signals from thermal gratings. Agreement between theory and experiment is exceptionally good. (c) 2000 Optical Society of America

OCIS codes: $\quad 000.2170,050.2770,120.6780,120.7280,190.4380$.
\end{abstract}

Laser-induced thermal acoustics (LITA, or laserinduced grating thermometry) is a four-wave mixing technique that has been successfully used for remote, nonintrusive, and instantaneous measurements of the speed of sound, thermal diffusivity, and fluid velocity. LITA is used by a number of laboratories for thermometry ${ }^{1-5}$ and for velocimetry applications with electrostrictive gratings ${ }^{6}$ and thermal gratings. ${ }^{7}$ A theoretical treatment of LITA can be found in Ref. 8 . Reference 9 extends the theory to include the effect of fluid velocities on homodyne-detected signals.

Heterodyne LITA velocimetry has been proposed ${ }^{8}$ as an alternative to laser Doppler anemometry for velocity measurements in luminous flows, flows with poor optical access, and flows with poor or no control of particulates. We report on the successful application of heterodyne LITA velocimetry with $\sim 0.5 \%$ uncertainty in the sound speed and velocity at Mach 0.1 from singleshot signals.

In LITA, two coherent intersecting pulsed laser beams create, by thermalization, electrostriction, or both, a density grating in the sample volume that evolves over time. A third, continuous interrogation laser beam, directed at the Bragg angle onto the sample volume, is scattered into a coherent signal beam whose intensity depends on the instantaneous density grating modulation depth. Convective motion of the laser-induced density grating along its normal vector produces a Doppler shift in the signal that is proportional to this velocity component. Heterodyne signal detection is employed to recover the Doppler shift. In this study we accomplish phase-sensitive detection by superimposing the signal beam and a reference beam onto a photomultiplier tube.

Experimental results are presented from heterodyne velocity measurements in a low-speed wind tunnel with thermal gratings in $\mathrm{NO}_{2}$-seeded air for a Mach number range $M=0-0.1$. Measurements are extracted from signals by optimal nonlinear filtering. The optimal nonlinear filter consists of a least-squares fit between a theoretical and an experimental signal. ${ }^{8}$ The theoretical signal used in the filter is an extension of the analysis in Ref. 9 for heterodyne detection with a finite frequency shift between the interrogation and the reference beams. Although it is not used in these experiments, such a shift (e.g., via a Bragg cell) could eliminate velocity ambiguity and improve the performance of the technique at low speeds.

Experiments are conducted in a closed-circuit wind tunnel with a test section diameter of $5 \mathrm{~cm}$. The tunnel is powered by a propeller mounted upon an airdriven motor ( $350 \mathrm{~W}$ at 6 bars) whose air supply and exhaust are piped through the wind tunnel wall to permit closed-circuit tunnel operation. The test gas is $\mathrm{NO}_{2}$-seeded air at atmospheric conditions.

The motor is driven by pressurized air from a blowdown reservoir that starts at 6 bars. Run times are approximately $60 \mathrm{~s}$, with speeds starting at $\sim 40 \mathrm{~m} / \mathrm{s}$ and decreasing with the reservoir pressure. Data are taken continuously during runs at the laser pulserepetition rate of $15 \mathrm{~Hz}$.

A standard forward-scattering focused-beam fourwave mixing geometry is employed. A frequencydoubled $Q$-switched Nd:YAG laser (Continuum Minilite II; $25 \mathrm{~mJ} /$ pulse, $3-5$-ns pulse duration) is used as the source for the grating excitation beams. Its beam is split into halves by a $50 / 50$ beam splitter. An arrangement of mirrors on kinematic mounts and translation stages directs the two beams with equal optical path length parallel onto a transmitter lens $(f=500 \mathrm{~mm})$, which focuses the beams onto an intersection point in the center of the test section. The beam crossing angle is $\sim 1.3^{\circ}$.

A cw argon-ion laser (Spectra-Physics Stabilite 2017 , at $488 \mathrm{~nm}$ ) provides the continuous interrogation and reference beams. A chopper wheel (Scitech 
Instruments optical chopper) produces $20-\mu \mathrm{s}$ pulses at 60 times the Nd:YAG laser's pulse repetition rate. Whereas these pulses are long compared with the density grating lifetime and hence can still be considered continuous during this time, the short duration of these pulses extends the linear operating range of the detector's photomultiplier tube. A 99/1 beam splitter divides the continuous beam into the interrogation and reference beams. A variable neutral-density filter provides intensity adjustments of the reference beam. Both beams are directed parallel and path-length matched onto the transmitter lens, which focuses them on the sample volume. The distance between the beams is adjusted for optimal phase matching. These beams intersect the plane of the two pulsed beams at an angle of $\sim 1.5^{\circ}$. A small fraction, $<0.01 \%$, of the interrogation beam scatters off the grating into a coherent signal beam. The reference beam is carefully aligned to follow the same path as the signal beam.

The timing and coordination of the lasers, data acquisition, and experiment are handled with a programmable logic and timing controller with 25-ns resolution (Labsmith LC880).

In the receiver, the signal-reference beams pass through an interference filter and are focused by a 40 -mm focal-length lens through a $200-\mu m$-diameter pinhole to suppress incoherently scattered and ambient light. A photomultiplier tube (Hamamatsu H5783-03; 0.65-ns response time) detects the signal, which is recorded onto a digital storage oscilloscope (Hewlett-Packard Infinium; four channels, $500 \mathrm{MHz}$, 2 Gsamples/s) and transferred to a Pentium II-based computer for storage and data analysis.

Each signal consists of a sequence of 1024 12-bit samples recorded at a sampling rate of $250 \mathrm{MHz}$. During each wind tunnel run, 1000 single-shot signals are recorded at $15 \mathrm{~Hz}$. The experimental traces are optimally filtered with a Levenberg-Marquardt-based ${ }^{10}$ least-squares fit to theory for which the flow velocity, the speed of sound, and the phase shift between the reference and the signal beams are fitting parameters. Other parameters, such as the beam diameters and the driver beam crossing angle, are determined in static calibration measurements.

Figure 1 shows examples of experimental heterodyne LITA signals and the best-fit theoretical signals. The theoretical signals are plotted with an offset for clarity. The agreement with theory is exceptionally good. Figure 1(a) was obtained before the wind tunnel was turned on; Fig. 1(b) represents signals from approximately the maximum flow velocity. The flow velocity decreases gradually from Fig. 1(b) to Fig. 1(f). The second-frequency component in the data from the Doppler shift of the signal beam is clearly evident.

Figure 2 shows velocity measurements (top trace) and sound-speed measurements (bottom trace) during a 60-s blow-down run of the wind tunnel. The inferred flow velocity before the wind tunnel is turned on is nonzero $(\sim 5 \mathrm{~m} / \mathrm{s})$ because of a signal distortion produced by small detector gain nonlinearities. This problem can be eliminated by the use of a frequency offset between the reference and the interrogation beams. The wind tunnel is not calibrated, and no quantitative statements about the accuracy can be made except in the case of zero flow velocity.

As uncertainty for the velocimetry data, the standard deviation of the velocity measurements about the linear regression plotted as a dashed line (with an offset for clarity) at the top of Fig. 2 is used. It is $\sim 0.2 \mathrm{~m} / \mathrm{s}$ and is independent of the flow velocity; i.e., the relative uncertainty decreases with increasing flow velocities. For the highest flow speeds $(\sim 40 \mathrm{~m} / \mathrm{s})$ the uncertainty is $0.5 \%$.

We use the standard deviation of the sound speed data about its mean as a measure of the uncertainty of the data, as the sound speed remains constant during the experiment. The uncertainty in the sound speed measurements is then $0.5 \%$.

Single-shot heterodyne LITA signals have been recorded in a wind tunnel. The theory, based on linear hydrodynamics and scattering, agrees well with
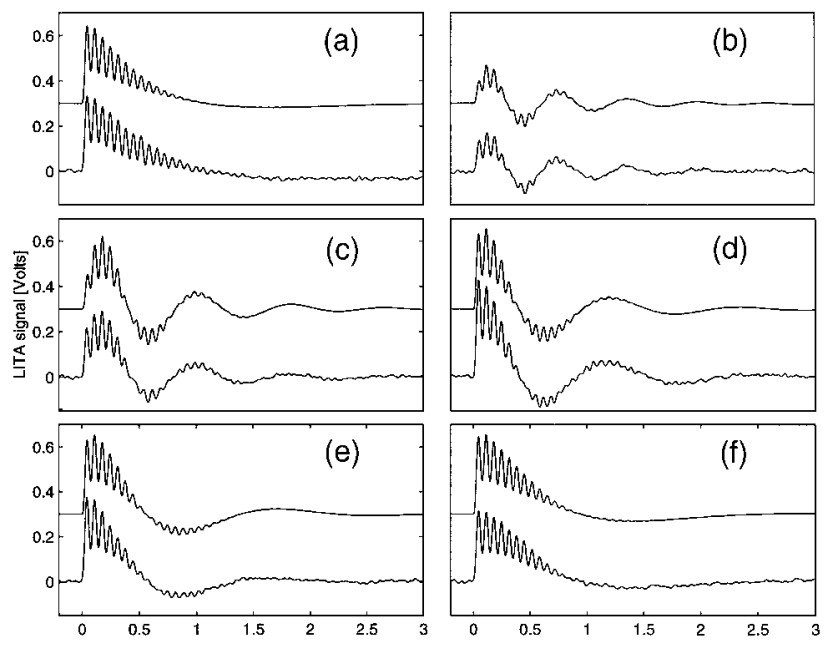

time [us]

Fig. 1. Experimental and theoretical heterodyne LITA signals for thermal gratings in atmospheric air. The theoretical signals are plotted with an offset for clarity. (a) $M=0.00$, (b) $M=0.11$, (c) $M=0.08$, (d) $M=0.06$, (e) $M=0.04$, (f) $M=0.02$.

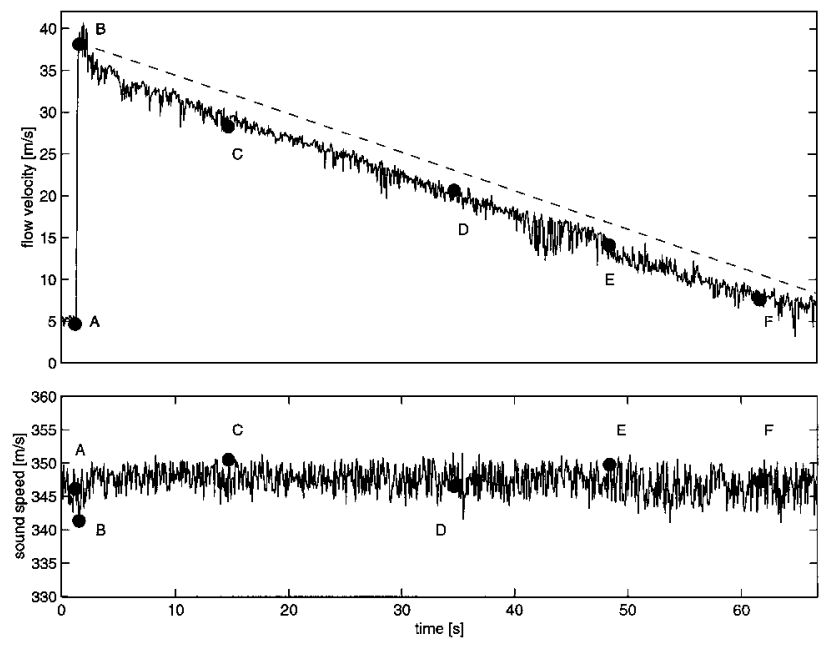

Fig. 2. Top, measured flow velocities during the blowdown experiment and bottom, values for the speed of sound. Each point labeled A-F corresponds to data labeled with the same letter but in lowercase in Fig. 1. 
the experimental signals. The signal lifetime sets a lower limit on the flow velocity that can be measured accurately. For example, in Fig. 1(f) the period of the Doppler shift is approximately the same as the signal lifetime. At low velocities, gain variations in the photomultiplier tube over the lifetime of the signals produce a large (consistent) systematic velocity error of $\sim 5 \mathrm{~m} / \mathrm{s}$. We can mitigate this error by characterizing the photomultiplier tube's response and removing the defects as best as possible from the signals. However, many effects produce low-frequency variations in a detected signal. Untreated, these effects produce flicker of $1 / f$ noise. Because of the practical impossibility of correcting for all these effects, we propose to use a Bragg cell in the path of the reference beam to shift its frequency by $O(100 \mathrm{MHz}$ ) (cf. typical local density approximation implementations). This frequency offset will appear in the signal, upshifted or downshifted by the Doppler frequency, depending on the direction of the motion of the fluid. By this upconversion, the frequencies of interest for velocity measurements leave the range in which $1 / f$ noise in the detection system is important for all practical velocities, eliminating large relative velocity errors at low speeds. The upconversion also removes ambiguity in the sign of the velocity measurement. The flow velocity and the speed of sound can then be measured with accuracies and uncertainties of $0.5 \%$ each over a wide velocity range.

Heterodyne LITA velocimetry should be applicable well into the hypersonic range of velocities. It has advantages compared with the local density approximation when optical access is limited, the flow is luminous, particulate content is not easily controlled, particle lag is excessive, and additional simultaneous information about the flow, such as the sound speed or thermal diffusivity, or their derived quantities the temperature and density, is desired. LITA velocimetry also produces measurements with microsecond temporal resolution, a factor in pulsed or highly unsteady flows. The disadvantages of the technique compared with the local density approximation are the additional complexity and the need for more-powerful lasers.

This study was supported by Advanced Projects Research, Inc., and by NASA Langley Research Center under NASA contract NAS1-99016. S. Schlamp's e-mail address is stefan@galcit.caltech.edu.

\section{References}

1. T. J. Butenhoff, Intl. J. Thermophys. 16, 1 (1995).

2. B. Hemmerling and D. N. Kozlov, Appl. Opt. 38, 1001 (1999).

3. M. S. Brown and W. L. Roberts, J. Propul. Power 15, 119 (1999).

4. R. C. Hart, R. J. Balla, and G. C. Herring, Appl. Opt. 38, 577 (1999).

5. S. Williams, L. A. Rahn, P. H. Paul, J. W. Forsman, and R. N. Zare, Opt. Lett. 19, 1681 (1994).

6. B. Hemmerling, D. N. Kozlov, and A. StampanoniPanariello, presented at the XVIII European CARS Workshop, Frascati, Italy, March 21-23, 1999.

7. D. J. W. Walker, R. B. Williams, and P. Ewart, Opt. Lett. 23, 1316 (1998).

8. E. B. Cummings, I. A. Leyva, and H. G. Hornung, Appl. Opt. 34, 3290 (1995).

9. S. Schlamp, E. B. Cummings, and H. G. Hornung, Appl. Opt. 38, 5724 (1999).

10. W. H. Press, Numerical Recipes in C: The Art of Scientific Computing (Cambridge U. Press, Cambridge, 1988). 